\title{
Tunable Anisotropic Strain in Laser Crystallized Silicon Core Optical Fibers
}

\author{
N. Healy ${ }^{1}$, S. Mailis ${ }^{1}$, N. M. Bulgakova ${ }^{2,3}$, T. D. Day ${ }^{4}$, P. J. A. Sazio ${ }^{1}$, J. V. Badding ${ }^{4}$ \\ and A. C. Peacock ${ }^{1}$ \\ ${ }^{I}$ Optoelectronics Research Centre, University of Southampton, Southampton, SO17 1BJ, UK \\ ${ }^{2}$ Institute of Thermophysics SB RAS, Novosibirsk 630090, Russia \\ ${ }^{3}$ HiLASE, Institute of Physics ASCR, 18221 Prague, Czech Republic \\ ${ }^{4}$ Department of Chemistry and Materials Research Institute, Pennsylvania State University, 16802 PA, USA
}

\begin{abstract}
A laser processing method for tuning the size of the anisotropic strain in a silicon core optical fiber is demonstrated. We propose that this technique can be used to modify the core's opto-electronic properties for applications with specific requirements.
\end{abstract}

\section{Introduction}

Silicon optical fibers represent a novel medium in which the large $\chi^{(3)}$ non-linearity of the core material can be exploited for signal processing and optical frequency generation in a geometrically standardized platform. However, silicon's opto-electronic functionality is subservient to its molecular structure, for example it has an indirect bandgap and has no $\chi^{(2)}$ non-linearity. In this paper we investigate a laser processing technique as a means to alter the core material's intrinsic properties so that its optical and electronic functionality can be tuned for targeted applications.

\section{Experiment}

Silica clad amorphous silicon (a-Si) core optical fibers with diameters of $1.7 \mu \mathrm{m}$ were fabricated using the high pressure microfluidic chemical deposition technique, see Fig. 1(a) [1]. The a-Si fibers were then crystallized using a focused $\mathrm{CW}$ argon-ion laser operating at $488 \mathrm{~nm}$ and a power of $2 \mathrm{~W}$. A set of programmable nano-positioning stages were used to scan the fibers through the radiation focus so that each position along the core was irradiated for a time controlled via the scan speed.

The resulting fibers were analyzed using a Raman spectrometer and the results for fibers prepared at two different irradiation times $\left(\mathrm{T}_{1}=0.5 \mathrm{~ms} \& \mathrm{~T}_{2}=500 \mathrm{~ms}\right)$ are shown in Fig. $1(\mathrm{~b})$. The difference in the peak position for each spectrum $\left(509 \mathrm{~cm}^{-1}\right.$ for $\mathrm{T}_{1} \& 519 \mathrm{~cm}^{-1}$ for $\left.\mathrm{T}_{2}\right)$ is a consequence of the strain induced by laser heating and indicates that the strain can be tuned as a function of irradiation time. The profile of the strain was measured using a synchrotron microfocus X-ray diffractometer and a 2-D diffraction pattern is shown in Fig. 1(c). The single Laue spot is a reflection from the <311> plane, and thus we can conclude that just one crystal is present at the sampled length The elongation of this spot is due to a variation of the crystal's lattice spacing which is indicative of an anisotropic strain that can be estimated to be $\sim 3 \%$. Such a large anisotropic strain can profoundly influence the core material's electrical and optical properties, for example, the electron mobility can be modified, the electronic bandgap can be shifted, or a sizable $\chi^{(2)}$ susceptibility may be induced. The effect that the anisotropic strain has on the optical properties of our silicon optical fibers will be discussed.

(a)

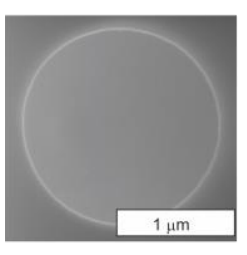

(b)

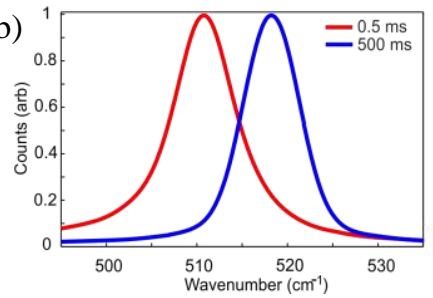

(c)

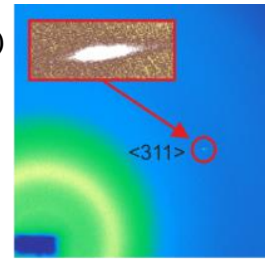

Fig. 1. (a) The cross-section of a silicon fiber. (b) The Raman spectra of 2 fibers exposed for $0.5 \mathrm{~ms}$ (red) and $500 \mathrm{~ms}$ (blue). (b) 2-D micro-focus $\mathrm{X}$-Ray diffraction pattern from the core of a strained silicon optical fiber; inset is a close-up of the elongated Laue spot.

\section{References}

[1] L. Lagonigro, N. Healy, J. R. Sparks, N. F. Baril, P. J. A. Sazio, J. V. Badding, and A. C. Peacock, "Low loss silicon fibers for photonics applications," Appl. Phys. Lett. 96, 041105 (2010). 